\title{
L'analyse séquentielle des parcours: pour une approche longitudinale et non causale des effets des interventions sociales
}

\section{Claire Ganne}

L'évolution des politiques sociales fait de la question des parcours un enjeu très actuel. L'affaiblissement des logiques catégorielles au profit d'approches plus transversales, puis davantage centrées sur l'individu et ses projets conduit les acteurs politiques et sociaux à s'intéresser aux parcours des personnes confrontées aux interventions sociales : parcours de soin, de vie, d'accompagnement... De manière plus globale, la multiplication des situations d'incertitudes ${ }^{1}$ et l'injonction faite aux individus de définir le sens et le cours de leur propre $v^{2}{ }^{2}$ mènent également à une plus grande prise en compte des parcours dans le champ académique, dans différentes disciplines des sciences humaines et sociales (en sociologie, en démographie, en sciences de l'éducation et en psychologie notamment).

Si elles ne sont pas nouvelles, les recherches et les études portant sur le devenir puis sur les parcours des sujets connaissent un développement important dans le champ scientifique et du côté des acteurs qui mettent en œuvre des politiques sociales (conseils départementaux, associations gestionnaires d'établissement...). C'est particulièrement vrai

Claire Ganne est maîtresse de conférences en sciences de l'éducation à l'université ParisNanterre, au sein de l'équipe Éducation familiale et interventions sociales auprès des familles (Cref-ea 1589). Ses travaux portent sur les centres maternels et parentaux et les parcours en protection de l'enfance.

1. Claude Dubar, « Sociologie. Les grands courants. », dans Encyclopaedia Universalis, Paris, Encyclopeadia Universalis, 2008.

2. Isabelle Astier et Nicolas Duvoux (sous la direction de), La société biographique : une injonction à vivre dignement, Paris, L'Harmattan, 2006. 
dans le secteur de la protection de l'enfance ${ }^{3}$. Dans ces contextes, l'étude des parcours ultérieurs est régulièrement envisagée comme un moyen d'évaluer les effets des interventions. Pourtant, comme le souligne Mary Ainsworth dès 1962 : «Dans les recherches étiologiques, surtout lorsqu'elles ont trait au développement de la personnalité, il s'écoule entre l'antécédent et l'effet une période relativement longue, parfois de plusieurs années, pendant laquelle les conditions ne sont pas contrôlées, la vie suivant son cours. Les recherches longitudinales portent sur la substance même de la vie et du développement humain, avec toutes les complications et les difficultés qui en découlent ${ }^{4}$. » Ce constat favorise l'adoption d'une posture compréhensive attribuant aux seuls acteurs le pouvoir de donner du sens à leur vécu, ce qui apporte des éléments de compréhension des processus mais ne permet pas toujours d'en mesurer la portée.

Quel statut donner alors au parcours dans la compréhension des effets des interventions ? Par quels outils les saisir ? Une troisième posture possible peut être celle du « positivisme narratif ${ }^{5}$ », inspirée par Andrew Abbott 6 , dont les travaux suscitent aujourd'hui fortement l'intérêt des chercheurs, comme en témoigne la publication récente du premier ouvrage de référence en français ${ }^{7}$. Sa pensée théorique et les outils qu'il déploie nous semblent particulièrement adaptés pour penser les liens entre interventions sociales et parcours ultérieurs, en s'inscrivant dans une approche qui prend en compte l'épaisseur temporelle et la dimension probabiliste des processus.

Il existe en effet une grande diversité d'épistémologies possibles face à la dimension longitudinale des phénomènes sociaux. Bessin, Bidart et Grossetti ${ }^{8}$ identifient ainsi quatre types de posture dans la prise en compte des événements en sociologie, qui peuvent d'ailleurs coexister chez un même auteur. Ces quatre postures sont scindées en deux groupes : deux postures qu'ils qualifient d'objectivantes et deux qu'ils qualifient

3. Nous renvoyons le lecteur qui voudrait approfondir ces questions aux revues de littérature suivantes : Isabelle Frechon et Annick-Camille Dumaret, «Bilan critique de 50 ans d'études sur le devenir adulte des enfants placés ", Neuropsychiatrie de l'enfance et de l'adolescence, vol. 56, n ${ }^{\circ} 3,2008$, p. 135-147 ; Sarra Chaieb, Revue de littérature. Les recherches francophones sur les parcours de placement, les transitions à l'âge adulte et le devenir des enfants placés, oned, 2013 ; ainsi qu'au récent numéro de Vie sociale : «Permettre la jeunesse. Ou comment accompagner les jeunes majeurs vers l'autonomie et l'indépendance», Vie sociale, $\mathrm{n}^{\circ} 12,2015$.

4. Mary D. Ainsworth, « Les répercussions de la carence maternelle : faits observés et controverses dans le contexte de la stratégie des recherches », Cahiers de santé publique-oms, $\mathrm{n}^{\circ} 14$, 1962, p. 104.

5. Marc Bessin, Claire Bidart et Michel Grossetti (sous la direction de), Bifurcations. Les sciences sociales face aux ruptures et à l'événement, Paris, La Découverte, 2010, vol. 1.

6. Andrew Abbott, Time Matters. On Theory and Method, Chicago, The University of Chicago Press, 2001.

7. Didier Demazière et Morgan Jouvenet (sous la direction de), Andrew Abbott et l'héritage de l'école de Chicago, Paris, Éditions ehess, 2016.

8. Marc Bessin et coll. (sous la direction de), op. cit., p. 32-33. 
de subjectivantes. Les deux postures objectivantes ont en commun de reconstruire l'objet à partir des informations disponibles : c'est le chercheur qui définit ce qui est important à observer. Mais ces deux postures se distinguent en revanche dans l'importance qu'elles accordent ou non à l'imprévisibilité, à l'événement, à la temporalité et au cours de la vie : une position déterministe classique cherchera à éliminer l'imprévisibilité de l'analyse, tandis qu'une posture plus narrative admettra l'existence d'événements imprévisibles pouvant produire des conséquences importantes. Les tenants d'approches « subjectivantes » ne s'accordent pas le droit de définir les temporalités, les événements significatifs, mais cherchent au contraire à saisir le sens que les acteurs donnent aux situations, soit dans une perspective compréhensive (comprendre le sens que l'acteur attribue aux événements de son parcours), soit dans une perspective uniquement discursive (seuls le discours et les changements de références qu'il présente sont objectivables et analysés).

Le matériel empirique sur lequel s'appuie cet article est issu d'une recherche doctorale en sciences de l'éducation ${ }^{9}$ portant sur les parcours d'enfants accueillis en centre maternel sept à huit ans après leur sortie, au cours de laquelle nous avons croisé une analyse quantitative des parcours et une perspective subjectivante du côté des acteurs, mères et enfants.

Après une discussion de différentes postures épistémologiques possibles dans l'analyse des parcours après une intervention sociale, repérables notamment dans les recherches sur le devenir ou le parcours en protection de l'enfance, nous nous appuierons sur cet exemple afin de montrer comment l'analyse séquentielle permet d'apporter des éléments quantitatifs de compréhension des processus qui ne soient ni uniquement descriptifs ni soutenus par une logique causale sous-jacente. Elle permet ainsi de comprendre en quoi les pratiques des professionnels de l'intervention socioéducative sont un "ingrédient ${ }^{10}$ » dans les processus de construction des parcours, sans s'enfermer dans la question de l'impact de l'intervention, quasiment insoluble en raison de l'interdépendance des dimensions et de l'importance de l'aléatoire dans les processus observés.

\section{ifférentes approches des Parcours}

L'étude du devenir des anciens placés fournit un bon exemple de l'évolution des postures méthodologiques et épistémologiques concernant la prise en compte des parcours. Si les démarches épidémiologiques ont été

9. Claire Ganne, Le devenir des enfants accueillis en centre maternel. Approche écologique du parcours et de la qualité de vie des enfants sept ans après la sortie d'un hébergement mère-enfant, thèse de doctorat en sciences de l'éducation, université Paris Ouest Nanterre La Défense, 2013.

10. Ariel Mendez (sous la direction de), Processus : concepts et méthode pour l'analyse temporelle en sciences sociales, Louvain-la-Neuve, Academia-Bruylant, 2010. 
privilégiées dans les premières recherches, elles ont ensuite atteint leurs limites, et les perspectives de recherche ont largement évolué vers la prise en compte de l'approche subjective des anciens placés, passant d'un regard rétrospectif sur la prise en charge ${ }^{11}$ à l'exploration du sens du parcours, mais également à l'évaluation du bien-être subjectif à l'âge adulte ${ }^{12}$ et à la construction de l'autonomie psychique et relationnelle ${ }^{13}$.

\section{Les limites de ladémarche épidémiologique dans l'étude des parcours après une inter vention sociale}

La démarche la plus ancienne et la plus répandue au regard du nombre d'articles s'y référant est celle de l'épidémiologie, que l'on peut définir comme « la distribution des maladies et des invalidités dans les populations humaines, ainsi que les influences qui la déterminent. Elle permet l'évaluation des méthodes de prévention (épidémiologie d'intervention) et l'observation de la répartition des maladies, permettant d'aller aux sources, puis aux causes (épidémiologie analytique, qui peut être scindée en recherche à visée explicative et recherche à visée pragmatique, ou prédictive) ${ }^{14} \gg$. Inscrite dans une recherche de lien de causalité entre passé et présent, elle a également été utilisée pour l'étude du devenir adulte des enfants marquées par une expérience sociale particulière : devenir des anciens placés, devenir des enfants pauvres... L'épidémiologie est alors comprise au sens large comme «la recherche de facteurs (leur rôle et leur répartition au sein d'une population représentative) dans l'expression d'une réalité qui est ici d'ordre social et d'ordre psychologique ${ }^{15}$ ».

Cette démarche a apporté des éléments de connaissances importants sur le devenir des sujets, et a notamment permis de battre en brèche l'idée communément répandue d'une reproduction intergénérationnelle massive du placement ${ }^{16}$. Cependant, elle présente également des limites lorsqu'il s'agit d'interpréter ces observations et de dépasser le simple stade descriptif. En effet, sur le plan explicatif, la perspective épidémiologique envisage essentiellement le devenir d'un individu comme un résultat (des thérapeutiques, des interventions, des politiques publiques),

11. Trudy Festinger, No One Ever Asked Us. A Postcript to Foster Care, New York, Columbia University Press, 1983.

12. Annick-Camille Dumaret, Émilie Guerry et Monique Crost, « Placements dans l'enfance et devenir à l'âge adulte. Insertion générale et qualité de vie liée à la santé », Neuropsychiatrie de l'enfance et de l'adolescence, vol. 59, n 5, 2011, p. 289-298.

13. Nadège Séverac et Pierre Moisset, "Au fondement de l'autonomie était un autre », Vie sociale, $\mathrm{n}^{\circ} 12,2015$, p. 129-148.

14. Daniel Oppenheim, « Devenir psychologique des enfants guéris d'une affection cancéreuse », Neuropsychiatrie de l'enfance et de l'adolescence, vol. 44, n 6-7, 1996, p. 291.

15. Michel Corbillon, Jean-Pascal Assailly et Michel Duyme, L'enfant placé. De l'Assistance publique à l'Aide sociale à l'enfance, Paris, ministère de la Solidarité, de la Santé et de la Protection sociale, 1990, p. 3.

16. Michel Corbillon, Jean-Pascal Assailly et Michel Duyme, «L'aide sociale à l'enfance. Descendance et devenir adulte des sujets placés », Population, vol. 43, n 2, 1988, p. 473-479. 
ce qu'illustre bien le terme « outcomes », traduit en français de manière peu satisfaisante par « résultats ». Son inscription dans une logique de causalité conduit à deux difficultés lorsqu'on observe les parcours après une intervention sociale : l'impossibilité de clôturer le système causal, et l'absence de groupe contrôle.

En effet, dès que le chercheur passe d'un projet descriptif à un projet explicatif fondé sur la recherche de liens de causalité, il se trouve confronté à la nécessité de respecter la clôture du système causal, c'est-à-dire la nécessité d'intégrer dans le modèle explicatif toutes les variables pouvant avoir une action sur l'objet observé ${ }^{17}$. Or, lorsque l'on s'intéresse à des caractéristiques structurantes de la vie des individus, et notamment lorsque l'on s'inscrit dans une perspective causale, cette procédure devient sans fin. Mais on ne peut modifier en permanence la stratégie de recueil des données ni complexifier le modèle à l'infini, au risque que celui-ci devienne ininterprétable. C'est la difficulté du raisonnement « toutes choses égales par ailleurs » en sciences humaines et sociales, alors que la réalité sociale et historique est toujours située et contextualisée. Les débats autour de l'impact direct de l'effet monétaire sur le devenir des enfants pauvres fournissent un bon exemple de ce type de difficultés. En effet, les recherches américaines classiques sur le devenir des enfants pauvres sont basées sur des modèles d'explication causale à plusieurs variables qui tentent de mesurer l'impact du revenu parental sur le devenir des enfants dans différents domaines ${ }^{18}$. Les auteurs cherchent à contrôler les facteurs exogènes qui pourraient biaiser l'observation, afin d'isoler l'effet du revenu des parents. Or la distinction entre facteurs endogènes et exogènes, qui permettrait de vérifier l'effet strict du revenu monétaire indépendamment d'autres facteurs, est particulièrement sujette à débat, et dépend bien sûr des modèles théoriques explicatifs adoptés par les différents auteurs ${ }^{19}$. Ainsi, le quartier d'habitation, parfois considéré comme un facteur exogène, est très dépendant des ressources financières des parents. Contrôler l'effet du quartier revient donc à minimiser l'impact du revenu sur le devenir. De la même façon, le niveau de stress parental, la santé mentale des parents, le niveau d'étude de la mère sont-ils réellement des facteurs exogènes dans l'observation des situations de pauvreté ?

\section{L'expérimentation sociale randomisée}

Dans une telle perspective causale, pour pouvoir attribuer un effet direct à un facteur, et notamment pour mesurer l'impact d'une intervention,

17. Michel Loriaux, «Des causes aux systèmes : la causalité en question », dans Robert Franck (sous la direction de), Faut-il chercher aux causes une raison? L'explication causale dans les sciences humaines, Paris, Vrin, 1994, p. 41-96.

18. Greg J. Duncan et Jeanne Brooks-Gunn, Consequences of Growing Up Poor, New York, Russel Sage Foundation, 1997.

19. Christine Bruniaux et Bénédicte Galtier, «L'étude du devenir des enfants de familles défavorisées : 1'apport des expériences américaine et britannique », Les papiers du CerC, 2003. 
l'idée se répand qu'il faudrait privilégier la mise en place de programmes d'expérimentation randomisée, comme sur le modèle des expérimentations cliniques dans le champ de la médecine : le bénéficiaire potentiel d'une intervention peut être affecté, par tirage au sort aléatoire, dans un groupe qui bénéficie de l'intervention ou dans un groupe contrôle qui bénéficie simplement des services habituels. Ces expérimentations dans le champ social montrent toutefois d'importantes limites sur le plan éthique et méthodologique dans le contexte sociopolitique français où les interventions ne sont pas pensées en termes de programme mais de service ${ }^{20}$. L'une des limites méthodologiques les plus importantes est la difficulté, pour les chercheurs, de maîtriser les critères d'inclusion ou d'exclusion des sujets dans le groupe expérimental, et donc de limiter les biais de sélection, car il s'agit d'un enjeu éthique et politique fort.

En tout état de cause, ces expérimentations ne pouvant conduire à priver des personnes d'un service déjà existant, elles ne peuvent être utilisées pour évaluer l'impact d'une intervention classique, mais uniquement des mesures expérimentales nouvellement créées. Néanmoins, elles répandent l'idée de la possibilité de mesurer l'impact des interventions sociales par l'observation du devenir ultérieur des sujets, en faisant l'économie de la compréhension des parcours de vie dans leur globalité.

Dans ce contexte, la tentation est grande, notamment pour les acteurs qui mettent en œuvre les politiques sociales, d'utiliser le parcours ultérieur pour mesurer les effets de l'intervention. Mais les conclusions restent toujours partielles et ambigües. Ainsi, l'analyse d'Isabelle Frechon concernant l'affirmation maintes fois reprise que $23 \%$ des jeunes sans domicile ont été placé durant leur enfance ${ }^{21}$ montre que ces résultats sont en partie dus à la porosité qui existe dans la construction même de ces deux catégories de l'action publique : un jeune bénéficiant d'un contrat jeune majeur et résidant en foyer de jeunes travailleurs passera mécaniquement de la catégorie d'enfant protégé à jeune sans domicile s'il se maintient dans ce foyer grâce à son salaire après la fin de son contrat jeune majeur ; d'autres jeunes étaient sans domicile avant leur prise en charge à l'Aide sociale à l'enfance... Ainsi, si l'on peut s'interroger sur la politique de protection de l'enfance concernant l'accompagnement de la transition à la sortie des dispositifs, il semble en revanche abusif de lire le lien entre situation de sans domicile et prise en charge à l'Aide sociale à l'enfance comme un lien de cause à effet.

20. Évelyne Serverin et Bernard Gomel, «L'expérimentation des politiques publiques dans tous ses états », Informations sociales, $\mathrm{n}^{\circ}$ 174, 11 février 2013, p. 128-137.

21. Isabelle Frechon, «Processus d'entrée et de sortie d'une situation de vulnérabilité. Le cas des "enfants placés" et des "sans-domicile" ", dans Maryse Bresson et coll. (sous la direction de), La vulnérabilité : questions de recherches en sciences sociales, Fribourg, Academic Press Fribourg, 2013, p. 107-122. 
Si la recherche de liens de causalité (fussent-ils probabilistes) entre intervention et parcours semble difficile, on peut faire le choix de cantonner les méthodes quantitatives à une dimension descriptive des phénomènes et de chercher à comprendre les processus au moyen de la perception subjective des acteurs. Pour Claude Dubar, « la modernité multipliant les situations incertaines, les chocs biographiques, les mobilités, les schémas déterministes ou actionnalistes ne sont plus pertinents. Plus aucun mécanisme régulateur, aucune co-construction du système et de l'acteur n'est assuré. Au contraire, c'est l'existence d'identités multiples d'acteurs et de systèmes très divers qui justifie le fait que ces nouvelles sociologies accordent une priorité à la mise en récit, par des auteurs, d'actions communes redéfinies comme des interactions structurantes ou des accomplissements pratiques $22 »$. Dans cette perspective, le discours narratif des acteurs ${ }^{23}$ est utilisé pour comprendre les composantes sociales d'une expérience personnelle, dégager des récurrences, des processus, des logiques d'action ${ }^{24}$. Ces démarches amènent des éléments de compréhension décisifs des processus du point de vue des acteurs. Néanmoins, elles n'ont pas pour ambition de décrire la portée quantitative de ces processus.

Il existe également une troisième possibilité : penser des recherches quantitatives sur les parcours qui ne soient pas cantonnées à une fonction purement descriptive, sans s'enfermer dans les difficultés épistémologiques et méthodologiques des méthodes qui fondent leur projet explicatif sur la recherche de liens de causalité. Les recherches longitudinales en cours sur la sortie de placement en France et au Québec s'inscrivent dans une préoccupation similaire : «Le cadre théorique dans lequel s'inscrivent nos deux projets ne se limite pas à l'évaluation des résultats (outcomes), il comprend également des approches conceptuelles complémentaires en appréhendant la pratique des acteurs situés dans leurs différents contextes [...] Dans cette perspective, les travaux sur l'acteur et sa marge de manœuvre permettent de prendre en compte le rôle du jeune qui interagit avec son environnement, en lui reconnaissant un pouvoir dans sa trajectoire et sa capacité à faire des choix, à partager son point de

22. Claude Dubar, « Sociologie. Les grands courants », op. cit.

23. Dans ce contexte, il faut d'ailleurs souligner la grande proximité, déjà largement relevée par ailleurs, de l'injonction de se raconter des politiques sociales, qui revendiquent également une évolution compréhensive, et de la demande biographique adressée par le chercheur (Jean-Paul Payet, Corinne Rostaing et Frédérique Giuliani (sous la direction de), La relation d'enquête : la sociologie au défi des acteurs faibles, Rennes, Presses universitaires de Rennes, 2010). Les personnes que le chercheur rencontre dans le cadre de récits de vie après une intervention sont pour certaines devenues des professionnelles du récit biographique institutionnel.

24. Daniel Bertaux, Le récit de vie, Paris, Armand Colin, 2010. 
vue sur les services qu'il reçoit et à accepter et refuser les soutiens, et ce, même si les contextes sociaux sont défavorables ${ }^{25}$. »

\section{Le positivisme narratif Abbott}

La proposition théorique et méthodologique d'Abbott porte justement sur la possibilité d'observer et de quantifier les phénomènes temporels dans une logique séquentielle et non causale. Abbott s'inscrit dans la poursuite des travaux des sociologues de l'école de Chicago, en particulier ceux d'Hughes sur les carrières. Il s'inspire aussi de la life course theory développée par le sociologue et psychologue Elder ${ }^{26}$, en la réinterprétant. Pour Abbott, les parcours de vie (comme d'autres éléments de la dynamique sociale) sont constitués de trajectoires et de turning points, qui sont « des changements courts entraînant des conséquences, qui opèrent la réorientation d'un processus ${ }^{27} »$. Ces moments de rupture viennent s'intercaler entre des séquences plus linéaires, rectilignes, qu'Abbott, à la suite d'Elder, appelle les trajectoires. Ces trajectoires sont conditionnées en partie par les structures sociales, de manière particulièrement forte aux deux extrêmes des catégories sociales. Les parcours de vie montrent des alternances de trajectoires régulières, relativement prévisibles, et de « chocs » internes ou externes qui réorientent le parcours et marquent le début d'une nouvelle séquence de trajectoire.

L'idée forte d'Abbott est que dans un parcours de vie, du point de vue de l'acteur, « les phases que l'on peut comprendre causalement semblent moins importantes que les phases qui sont incompréhensibles d'un point de vue causal ${ }^{28} »$. Il montre en effet que les relations de causalité et de structure expliquent bien les épisodes de vie qui correspondent à des trajectoires particulièrement programmées par des contraintes structurelles, alors que pour l'acteur lui-même, les turning points, les moments de bifurcation qui affectent plusieurs dimensions de la vie, ont des conséquences bien plus importantes pour comprendre son parcours de vie. Cette inversion logique apparente le conduit à attribuer un pouvoir plus important aux effets de séquences qu'aux corrélations pour comprendre les processus sociaux, le lien entre le passé et le présent et entre les structures sociales et les individus. Cette idée nous semble particulièrement féconde pour analyser les parcours dans le cadre des interventions sociales.

25. Martin Goyette et Isabelle Frechon, « Comprendre le devenir des jeunes placés : la nécessité d'une observation longitudinale et représentative tenant compte des contextes socioculturel et politique », Revue française des affaires sociales, $\mathrm{n}^{\circ}$ 1-2, 15 juillet 2013, p. 164-180.

26. Glen H. Elder, «The life course and human development », dans Handbook of Child Psychology $5^{\text {th }}$ Edition, volume 1 : Theoretical Models of Human Development, New York, John Wiley \& Sons, 1998, p. 939-991.

27. Andrew Abbott, « À propos du concept de turning point », dans Marc Bessin et coll. (sous la direction de), op. cit., p. 207.

28. Ibid., p. 196. 
Pour Abbott, les raisonnements sociologiques liant des variables entre elles ont conduit à créer une « réalité linéaire générale » complètement déconnectée des phénomènes sociaux réels. L'approche séquentielle des faits sociaux qu'il propose fonde son projet épistémologique sur la volonté de réintroduire le temps dans toute son épaisseur dans les analyses des sciences sociales et conduit à prendre en compte les éléments écologiques du contexte pour comprendre et expliquer les processus. Il a donc cherché à introduire dans les sciences sociales des outils méthodologiques permettant de développer les analyses quantitatives longitudinales des parcours, comme l'analyse de séquences ${ }^{29}$.

L'introduction de ces méthodes d'analyse de séquence dans les sciences sociales repose sur l'idée que la dynamique sociale est un processus temporel impossible à saisir par des mesures qui « aplatiraient » trop le temps et les systèmes. Il s'agit une conception interactionniste des modalités d'action des structures sociales, qui amène à prendre en compte les différentes écologies ${ }^{30}$ au sein desquelles un même événement peut être interprété. Selon Abbott, le monde social change sans arrêt et ce n'est que parce qu'une grande partie de la structure sociale se reproduit en permanence que nous avons une impression de durée et d'immobilité. Ce sont les relations entre les acteurs sociaux qui expliquent ce phénomène de reproduction permanente et ce sont ces réseaux de relations et d'actions reproduites que nous nommons structure : «Le passé est encodé dans le présent sous la forme d'agencements de connexions que nous appelons structures. La production du prochain moment de la vie sociale se fait sur la base produite par cette structure. Et les agencements de structures laissent toujours des ouvertures pour des actions qui, si elles sont ajustées à la situation, peuvent changer assez rapidement la plus durable des structures $^{31}$.»

Il s'agit finalement de passer d'une approche par les variables à une approche par les configurations : en repérant les récurrences, les agencements locaux de données, on arrive à une forme de description, mais également de compréhension des ingrédients d'un processus, sans s'emprisonner dans la recherche abstraite de l'effet et de la cause, mais en reconnaissant que le sens des événements dépend grandement de leur localisation (au sein d'une biographie, d'une interaction).

29. Andrew Abbott et Alexandra Hrycak, « Measuring resemblance in sequence data. An optimal matching analysis of musicians' careers ", American Journal of Sociology, vol. 96, $\mathrm{n}^{\circ} 1,1990$, p. 144.

30. Pour Abbott, les écologies sont des structures multiples et interdépendantes. Le monde social tout entier peut être envisagé comme un ensemble d'écologies multiples et liées entre elles : écologie d'une profession, d'un État, d'une ville : chaque système est en interaction avec les autres, et les événements qui surviennent peuvent s'inscrire dans plusieurs systèmes écologiques à la fois. Le mot écologie désigne ici une structure sociale moins unifiée que ne l'est une machine ou un organisme, mais beaucoup plus solidaire, unifiée, que ne le sont les unités atomiques du libéralisme classique.

31. Andrew Abbott, « À propos du concept de turning point », op. cit., p. 206-207. 
Quelles que soient les disciplines, la prise en compte du temps et des interactions au sein des systèmes est intrinsèquement liée dès que l'on tente de comprendre et d'observer des processus et non des corrélations. Lorsque l'on redonne de l'épaisseur au temps, on ne peut manquer de s'intéresser aux contextes et aux différents systèmes qui entourent l'individu. Et si la prise en compte du temps passe par celle des systèmes, l'inverse semble être vrai aussi : lorsque l'on s'intéresse à une analyse des systèmes, on est amené à redonner de l'épaisseur au temps, comme l'illustrent les évolutions du modèle écologique du développement humain, d'après Urie Bronfenbrenner ${ }^{32}$. Ce modèle pour comprendre le développement de l'enfant est très influencé par la pensée systémique. Il postule que le développement se produit grâce à des interactions prolongées de l'enfant (ou de l'adulte) avec les systèmes qui l'entourent : le microsystème au sein duquel il interagit directement; le mésosystème constitué par les relations entre ces différents microsystèmes ; l'exosystème qui peut avoir des conséquences par le biais de son action sur l'un des acteurs d'un microsystème ; et le macrosystème constitué par l'ensemble des valeurs, des normes et des lois qui structurent la société dans laquelle ces différents systèmes se développent. L'ensemble de ces systèmes forme l'écosystème : celui-ci est composé de différents ensembles interactionnels, « emboîtés » les uns dans les autres, « comme des poupées russes » selon l'expression de Bronfenbrenner.

Au fil de ses différents travaux, Bronfenbrenner accorde une importance croissante aux processus comme moteur du développement, qui conduira à l'introduction du concept de chronosystème ${ }^{33}$, et à une nouvelle formulation du modèle, intitulé alors modèle Processus-personne-contexte-temps ${ }^{34}$. La prise en compte de la temporalité ne fera que se renforcer au fil du temps.

Ainsi, le cadre théorique de l'écologie du développement humain, largement utilisé pour penser le développement de l'enfant dans ses différents contextes de vie, s'est construit sur un point de départ très différent (comprendre le développement de l'enfant) mais présente finalement des similitudes avec la pensée d'Abbott. Ces approches ont en commun de s'intéresser au temps, aux processus, aux interactions, aux systèmes, davantage qu'aux corrélations et à la causalité.

32. Urie Bronfenbrenner, The Ecology of Human Development. Experiments by Nature and Design, Cambridge-Londres, Harvard University Press, 1979.

33. Urie Bronfenbrenner, «Ecology of the family as a context for human development. Research perspectives », Developmental Psychology, vol. 22, nº 6, 1986, p. 723-742.

34. Urie Bronfenbrenner, « Le modèle Processus-personne-contexte-temps », dans Le modèle écologique dans l'étude du développement de l'enfant, Sainte-Foy, Presses de l'université du Québec, 1996, p. 11-59. 
En effet, l'approche de Bronfenbrenner s'est également constituée en rupture par rapport à la place accordée à la causalité et aux relations linéaires entre les variables dans l'étude des phénomènes humains. Pour lui, l'utilisation de stratégies d'analyse qui ne considéraient que les relations linéaires entre les variables a considérablement ralenti la recherche sur le développement et amène à croire que les processus proximaux seraient universels et équivalents quels que soient les personnes et les contextes de vie ; son modèle tente de remettre au centre les processus et les contextes ${ }^{35}$. D'ailleurs, Elder estime que la théorie des parcours de vie a plusieurs racines, dont la sociologie interactionniste et ses travaux sur les carrières, mais aussi la psychologie du développement et notamment les approches écologiques ${ }^{36}$. De son côté, Bronfenbrenner affirme que les travaux d'Elder ont largement influencé les remaniements du modèle écologique, notamment avec l'importance nouvelle accordée au temps ${ }^{37}$.

Bronfenbrenner propose de plus que la théorie des parcours de vie d'Elder s'intéresse également aux effets retours sur les processus historiques : le développement au fil d'un parcours de vie n'est pas uniquement produit par le changement historique, il est lui-même producteur de ce changement. La manière dont les individus d'une société donnée se développent porte en germe les évolutions sociales, historiques et politiques à venir. Cette idée entre en correspondance avec le changement permanent, masqué par la reproduction permanente des structures sociales par les acteurs de la conception d'Abbott. Ainsi, la structure sociale dans laquelle s'inscrit le développement d'un enfant, ou d'un être humain en général, n'est pas un simple cadre, une simple toile de fond, mais elle est constitutive du processus développemental, elle est prise elle-même dans ce processus.

Quelle que soit la perspective théorique choisie, l'analyse du devenir se situe au confluent des approches centrées sur le sujet ou sur les structures sociales. Les effets de structure, s'ils sont présents, n'expliquent jamais totalement un parcours de vie, qui ne reste que probable. Le rapprochement des travaux d'Abbott et de Bronfenbrenner permet de concevoir le développement humain comme un processus écologique et séquentiel. Malgré cette remise en question des analyses causales utilisées classiquement dans certains courants de la psychologie et de la sociologie, et contrairement à d'autres courants théoriques, sur le plan opérationnel Bronfenbrenner comme Abbott ne réfutent pas l'utilisation de méthodes quantitatives au profit de méthodes uniquement qualitatives, mais proposent l'utilisation d'outils quantitatifs avec d'autres finalités que

\section{Ibid.}

36. Glen H. Elder, Monica K. Johnson et Robert Crosnoe, « The emergence and development of Life Course Theory », dans Handbook of the Life Course, New York, Springer, 2004, p. 3-19. 37. Urie Bronfenbrenner et Pamela A. Morris, « The ecology of developmental processes », dans Handbook of Child Psychology $5^{\text {th }}$ Edition, vol. 1, op. cit., p. 993-1027. 
la recherche de corrélations linéaires qui seraient le reflet de relations de causalité : construction du devis de recherche en faisant intervenir l'ensemble des éléments du modèle chez Bronfenbrenner et développement d'outils spécifiques d'analyse séquentielle chez Abbott, que nous allons maintenant illustrer.

\section{Quelques exemples d'utilisation de l'analyse séquentielle des trajectoires: le cas des enfants accueillis en centre maternel avec leur mère}

Nous allons maintenant nous attacher à décrire la mise en application possible de ce cadre théorique, pour illustrer le type de résultats qu'il est susceptible de produire, à partir du matériel empirique recueilli dans le cadre d'une thèse de doctorat en sciences de l'éducation portant sur les parcours d'enfants après un accueil en centre maternel ${ }^{38}$.

\section{Matériel empirique mobilisé}

La population initiale de la recherche est constituée de l'ensemble des familles sorties en 2002 ou en 2003 d'un centre maternel, dans deux départements, soit 315 familles au total. Lors de l'enquête, les familles avaient quitté les établissements depuis six à sept ans, et les enfants étaient âgés de 6 à 11 ans.

Le recueil de données s'est déroulé en plusieurs étapes, associant une démarche quantitative reconstituant le maximum de parcours ultérieurs, concernant l'évolution des mesures de protection et l'évolution des situations familiales et résidentielles, avec une démarche qualitative s'intéressant en profondeur à un nombre plus restreint de mères et d'enfants, afin d'appréhender les processus de construction du parcours et leur perception de leur situation actuelle. La reconstitution des parcours ultérieurs a été effectuée au moyen d'une étude des dossiers de l'Aide sociale à l'enfance et d'une enquête par questionnaire biographique, menée en face à face auprès des mères retrouvées acceptant de participer à la recherche. Les deux sources d'information ont été croisées, ce qui a permis de reconstituer 197 parcours résidentiels et en protection (63\% de la population initiale). Ces données ont été approfondies grâce à des entretiens biographiques menés avec 49 mères, et à des entretiens et des questionnaires de qualité de vie réalisés auprès de 33 enfants.

38. Établissements hébergeant des femmes enceintes ou accompagnées d'enfants de moins de 3 ans dans le cadre de la protection de l'enfance. 
Il ne s'agira pas ici de présenter les résultats de ce travail, qui sont pour certains disponibles par ailleurs ${ }^{39}$, mais plutôt d'illustrer ce que permet ce cadre théorique : une description quantitative des parcours qui n'écrase pas le temps, une mise au jour des ingrédients des processus de construction des parcours et des processus à l'œuvre durant les accompagnements. L'utilisation du cadre théorique d'Abbott produit ainsi des résultats intéressants du fait des techniques méthodologiques qui lui sont associées pour décrire la réalité, mais également du fait de la conception des logiques temporelles qui le sous-tend.

En effet, cela permet non seulement de décrire les parcours de manière nouvelle mais aussi d'en proposer une interprétation qui ne renonce pas à observer le lien entre interventions sociales et parcours de vie, s'en s'enfermer dans la recherche de causalité. Il permet ainsi de contribuer à une meilleure compréhension des liens entre parcours ultérieurs et interventions sociales, sans tenter de réduire le devenir des personnes au résultat des interventions.

\section{Décrire des typologies de trajectoires sans écraser le temps}

Le premier intérêt, sur le plan des techniques de traitement des données, est de disposer d'outils descriptifs des parcours dans le temps qui permettent d'identifier des typologies de trajectoires, et de décrire ainsi des configurations existantes sans en écraser la dimension temporelle.

Dans la recherche menée sur les parcours après la sortie d'un centre maternel, lareconstitution des parcours résidentiels et en protection après la sortie a permis d'analyser près de 200 parcours. Les données recueillies prennent la forme de trajectoires familiales, d'une durée de huit à neuf ans, au cours desquels peuvent se succéder un nombre variable de situations résidentielles et de mesures de protection de l'enfance. Afin de dégager une intelligibilité de cette accumulation de trajectoires, nous avons utilisé les outils issus de l'analyse de séquences, et notamment les Méthodes d'appariement optimal (mao), introduites par Abbott en sociologie. Les méthodes d'appariement optimal (optimal matching) ont été développées dans les années 1950 en informatique, puis utilisées dans des disciplines variées, notamment en biologie. Il s'agit d'un ensemble de techniques statistiques permettant d'exploreret decomparerdes suites d'objets (des séquences d'adn, des étapes dans une carrière professionnelle...). Cestechniques permettent de calculer des distances deux àdeux entre l'ensemble des séquences, qui sont ensuite regroupées en cluster,

39. Claire Ganne et Geneviève Bergonnier-Dupuy, «Trajectoires résidentielles et interventions socio-éducatives. L'exemple des familles accueillies en centre maternel », Les Sciences de l'éducation pour l'ère nouvelle, vol. 45, nº 3, 2012, p. 103-128 ; Claire Ganne, «Après la sortie d'un centre maternel : l'impact des trajectoires sur la qualité de vie des enfants », Revue française des affaires sociales, $\mathrm{n}^{\circ}$ 1-2, 15 juillet 2013, p. 96-121. 
en minimisant au maximum les distances au sein d'un même groupe. On obtient ainsi des typologies de séquences permettant de décrire les trajectoires observées ${ }^{40}$.

L'intérêt de ces techniques pour l'étude des parcours institutionnels est manifeste, dans la mesure où les recherches antérieures ont largement montré que les parcours des enfants concernés par une mesure de protection de l'enfance pouvaient être des parcours de placement, de déplacement, de replacement ${ }^{41} \ldots$ Ainsi, dans l'un des deux départements enquêtés, $13 \%$ des enfants font l'objet d'un placement (32/166) au moment de la sortie du centre maternel. Toutefois, près de la moitié des enfants enquêtés connaissent par la suite une ou plusieurs mesures de protection de l'enfance dans la suite de leur parcours (77/166). Ces enfants peuvent être répartis en trois groupes : 28 enfants connaissent une trajectoire dominée par des mesures de suivi en milieu ouvert, avec des alternances de périodes sans mesures ; 28 autres enfants sont accueillis dans le cadre de placements longs à l'Aide sociale à l'enfance, sans retour au domicile de la mère ; enfin, 21 enfants connaissent des trajectoires complexes, composées d'allers-retours entre le domicile de la mère, souvent accompagnés de mesures en milieu ouvert, et de mesures de placement judiciaire. Or il faut souligner que plus du tiers des enfants qui connaitront des placements longs ne sont pas placés au moment de la sortie, mais dans les deux années qui suivent (11/28). Inversement, le tiers des enfants placés à la sortie du centre maternel regagnent le domicile de leur mère dans les années qui suivent (11/32, avec une stabilité de ce retour pour 9 d'entre eux pendant les six années suivantes).

Caractériser les parcours des enfants par leur situation à un moment donné du temps (que ce soit au moment de la sortie du centre maternel, quelques mois après, ou quelques années après) ne permet donc pas d'identifier des catégories de situations pertinentes, et l'utilisation d'outils prenant en compte l'évolution des situations au fil du temps est nécessaire. De plus, en croisant les dimensions considérées dans les parcours des individus, on parvient à identifier des configurations de situations qui permettent d'approcher une plus grande intelligibilité des parcours. Sur le plan technique, l'analyse de séquence d'état permet une identification de la portée des phénomènes et ainsi une identification des configurations pertinentes. Cette grille de lecture peut également être employée pour analyser des situations en plus petit nombre, en identifiant les ingrédients des processus tant dans le parcours après la sortie que pendant un accompagnement.

40. Pour plus d'informations en français, voir Thibaut de Saint-Pol et Laurent Lesnard, « Introduction aux méthodes d'appariement optimal (Optimal Matching Analysis) », Bulletin de méthodologie sociologique, $\mathrm{n}^{\circ}$ 90, 2006, p. 5-25.

41. Émilie Potin, Enfants en danger, enfants protégés, enfants sécurisés ? Parcours de (dé) placement(s) des enfants confiés à l'Aide sociale à l'enfance, thèse de doctorat, université de Bretagne occidentale, 2009. 
L'aspect heuristique d'une conception séquentielle et écologique des parcours ne réside pas uniquement dans l'emploi de techniques descriptives longitudinales, mais bien dans la compréhension des processus de construction des parcours qu'elle permet : en analysant les enchaînements d'événements, on constate des récurrences, qui ne sont pas interprétées de manière causale mais de manière narrative.

À titre d'exemple, les 66 situations pour lesquelles nous disposions d'un questionnaire biographique permettaient d'analyser l'interaction d'un bien plus grand nombre de dimensions au fil du parcours. Nous avons alors tenté de décrire les trajectoires menant à différents types de mesures de protection de l'enfance, en synthétisant les interactions entre les dimensions résidentielles, familiales et scolaires que nous avons pu observer. Bien sûr, ces mesures de protection sont souvent décidées en fonction de l'observation des processus proximaux, sur lesquels nous n'avons pas centré notre recherche. Néanmoins, l'observation de récurrence dans les configurations invite à reconnaître que certains contextes favorisent des types de décision de protection. Cela peut être dû au fait que ces contextes soutiennent ou au contraire fragilisent les processus proximaux, mais également aux critères intervenant dans l'évaluation des situations d'enfance en danger selon les réalités départementales. On observe finalement un processus qui fait intervenir la trajectoire résidentielle, familiale et scolaire, les caractéristiques de la mère, ses éventuelles difficultés de santé et le département de prise en charge ${ }^{42}$.

Dans notre population, l'un des premiers éléments déterminants est la présence ou l'absence d'un logement stable pour la mère et l'enfant. Si la mère vit en couple et que la famille dispose d'un logement stable, c'est ensuite la violence du conjoint qui fera la différence : en présence d'un conjoint violent, l'enfant connaît une très forte probabilité de faire l'objet d'un placement judiciaire ; dans le cas contraire, les enfants vivant avec deux parents (qu'il s'agisse du père biologique ou d'un beau-père) ne font pas l'objet de mesure de protection. Si la mère ne forme pas de nouvelle union, des mesures de protection administrative ou en milieu ouvert sont davantage susceptibles d'apparaître : accueil provisoire à la demande de la mère en cas de difficultés de santé, ou mesures de suivi en milieu ouvert et interventions de techniciennes de l'intervention sociale et familiale si l'enfant manifeste des difficultés qui alertent l'école.

42. Il faut souligner que cette modélisation des enchainements séquentiels menant à une mesure de protection reste tout à fait spécifique à notre population, définie dès le départ par la conjonction de la précarité résidentielle et de la monoparentalité, au moins temporaire, et que cela ne s'appliquerait sans doute pas de la même manière à d'autres publics suivis en protection de l'enfance. 


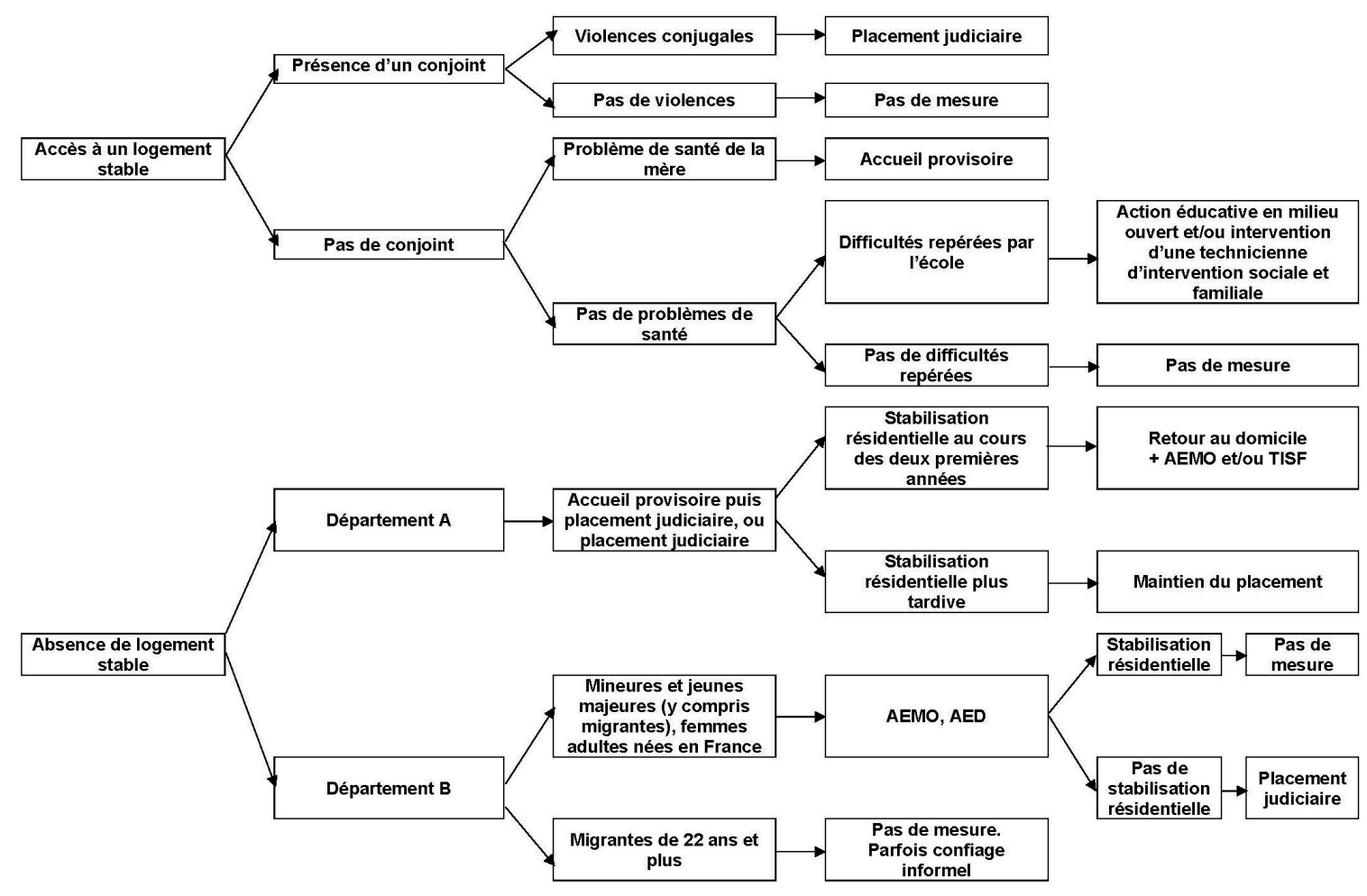


En l'absence de logement stable, la situation sera très différente. Les trajectoires observées dans les deux départements se différencient nettement. Il faut sans doute voir là un effet transactionnel entre les caractéristiques du public accueilli dans les deux départements et des pratiques sociales ancrées dans une histoire territoriale et une offre départementale. Ainsi, lorsqu' une femme et son enfant se retrouvent en situation d'errance à la sortie du centre maternel, la probabilité que ce dernier soit placé est beaucoup plus importante dans le département A que dans le département $\mathrm{B}$. La possibilité pour la mère d'accéder à une stabilité résidentielle va être déterminante sur la suite du parcours, puisque certains enfants vont revenir au domicile de la mère alors que d'autres vont connaître des trajectoires de placement long.

Dans le département B, en début de parcours, le maintien de l'enfant auprès de sa mère semble privilégié, $\mathrm{y}$ compris dans des conditions précaires. Dans ce département, les caractéristiques de la mère ont un impact fort : ainsi, dans les trajectoires que nous avons observées, les enfants des mères migrantes adultes ne font quasiment jamais l'objet de mesure de protection, bien que certains d'entre eux connaissent des conditions matérielles de vie particulièrement difficiles durant plusieurs années. Dans ce cas, c'est parfois la mère elle-même qui met en œuvre une situation de confiage informel. Les enfants des femmes plus jeunes (qui dans ce département sont nombreuses à avoir connu une situation de migration durant l'enfance ou l'adolescence) ou nées en France font en revanche plus souvent l'objet de mesure de suivi en milieu ouvert. Si la situation résidentielle se stabilise, la mesure est levée ; à l'inverse, si la situation perdure, un placement pourra être décidé.

Pour les familles sans domicile, il existe donc un glissement temporel dans l'ordre des séquences entre les deux départements, au moins pour les enfants des mères les plus jeunes : dans le département $B$, la mesure de milieu ouvert intervient avant le placement ou la stabilisation résidentielle, alors qu'elle intervient après ces deux événements dans le département $\mathrm{A}$. Le maintien à long terme d'une instabilité résidentielle entraîne par contre un risque fort de placement dans les deux départements.

L'ensemble des dimensions du parcours sont particulièrement intriquées. Ainsi, dans un contexte structurellement contraint par la difficulté d'accès au logement pour des familles monoparentales à faibles ressources, la trajectoire résidentielle et familiale de l'enfant va dépendre à la fois de la trajectoire conjugale de sa mère et des capacités de celle-ci à mobiliser des soutiens professionnels. En retour, les conditions matérielles de vie (logement autonome ou hébergement, qualité du logement, quartier d'implantation...) ont un impact sur la dynamique familiale et peuvent contribuer à la renforcer ou à la fragiliser. Ce phénomène est d'ailleurs particulièrement visible pour les femmes dont les enfants ont été placés au cours de la trajectoire ultérieure, qui auront à stabiliser leur situation tant 
sur les dimensions résidentielles que familiales et conjugales. Cela pénalise finalement encore plus les femmes victimes de violences conjugales, qui se remettront moins souvent en couple que les autres et accèderont moins souvent à un logement autonome stable. Ainsi, les bifurcations résidentielles et familiales de la trajectoire de l'enfant résultent d'interactions complexes, que l'utilisation d'une logique narrative donne bien à voir.

\section{Comprendre les processus en cours d'accompagnement}

Enfin, la recherche des ingrédients du processus et des séquences temporelles, en prenant en compte le sens des événements dans les différents systèmes concernés, est également intéressante pour comprendre les processus dans le cours même de l'intervention sociale. En centre maternel, le développement des enfants constitue un enjeu important entre les mères et les professionnels, puisque leurs missions comprennent explicitement le soutien à la relation mère-enfant et la protection de ce dernier. Dans la mesure où il s'agit d'un enjeu identitaire essentiel, il est très délicat pour ces femmes d'accepter une intervention centrée sur la relation aux enfants.

L'analyse de 14 situations, en croisant le cadre Processus-personnecontexte-temps et une reconstitution séquentielle des événements ayant jalonné l'accompagnement de la famille en centre maternel, met en lumière le lien fort qui existe entre l'évaluation de la relation mère-enfant et la qualité de la relation entre femmes et professionnels. Ce lien ne fonctionne pas à sens unique. En effet, la reconstitution chronologique des événements ${ }^{43}$ met en évidence un processus interactif : lorsque les relations entre les femmes et les professionnels se tendent pour d'autres raisons (fuite du suivi éducatif, place du père, non-respect du règlement...), les rapports sociaux semblent se focaliser davantage sur les points à surveiller dans le développement de l'enfant, initiant un processus d'emballement des relations qui aboutit parfois à des blocages voire à des sorties du centre maternel. On peut en particulier constater que pour l'ensemble des enfants dont l'évolution est jugée préoccupante, la relation entre la mère et les travailleurs sociaux est marquée par une non-adhésion à la prise en charge ou par une dégradation des relations au fur et à mesure du séjour.

Il est parfois possible, du fait de la chronologie des événements, de tenter de comprendre quelle relation a impacté l'autre. Ainsi, pour une situation, la dégradation des relations entre la mère et les travailleurs sociaux suite à un changement de référent paraît entrainer une focalisation

43. Dans une perspective objectivante, les seuls entretiens rétrospectifs ne permettent pas de reconstituer cette ligne temporelle. En revanche, les dossiers sociaux, produits dans un contexte discursif particulièrement contraint, portent les traces d'événements survenus au cours de l'accompagnement : les rapports étant rédigés de six mois en six mois, on peut repérer l'ordre d'apparition des thèmes (rendez-vous manqué, désaccord entre mère et professionnel, apparition de questionnements sur le développement de l'enfant...) dans une logique temporelle. 
du regard des professionnels sur les points de développement à surveiller, ce qui ne fait que renforcer le conflit entre la mère et le nouveau référent. Dans un autre cas, la dégradation des relations semble se cristalliser autour de la sortie, la femme ayant le sentiment d'être insuffisamment soutenue dans sa recherche de logement par les travailleurs sociaux, et le faisant savoir avec agressivité. Cette dégradation des relations entraîne la proposition d'une mesure d'aide éducative à domicile à la sortie, qui n'avait pas été envisagée avant le conflit opposant la mère et les travailleurs sociaux, alors que l'évolution de l'enfant et la relation mère-enfant étaient évaluées de la même manière avant le conflit.

Les situations de non-renouvellement de prise en charge pour nonadhésion au suivi sont particulièrement symptomatiques d'un emballement des dynamiques relationnelles du système. Ainsi, l'une des femmes verra sa relation se dégrader au fil du séjour avec les professionnels. En effet, d'après les travailleurs sociaux, la mère est en difficulté pour gérer l'opposition de son fils, qui devient de plus en plus provocateur et agité avec le temps. De son côté, elle se dit surtout inquiète pour sa fille aînée, confiée en Côte d'Ivoire, et elle ne comprend pas l'inquiétude des professionnels concernant son fils accueilli avec elle, qui se développe à son rythme. Lors d'une réunion de synthèse à l'Aide sociale à l'enfance, le séjour est renouvelé pour trois mois, avec injonction faite à Madame de solliciter des rendez-vous avec sa référente. Après cette synthèse, Madame se conforme à cette injonction et sollicite des rendez-vous, mais, selon les professionnels, « il est impossible de travailler autour de la relation mère-enfant. Madame estime qu'elle n'en a pas besoin ». De son côté, Madame exprime des demandes matérielles, notamment concernant l'accès au logement. Au vu du contenu du travail éducatif, l'établissement et l'Aide sociale à l'enfance décident de mettre fin au séjour. La famille est alors hébergée chez une amie de Madame, sans qu'un relais ou un suivi puisse être mis en place alors que les professionnels restent inquiets pour l'évolution de son fils. Ainsi, Madame se conforme aux injonctions de l'Aide sociale à l'enfance pour continuer à bénéficier de l'hébergement mais résiste au suivi socioéducatif focalisé sur le lien mère-enfant. Pour elle, ses besoins pour remplir son rôle de mère sont d'une autre nature : accéder à un titre de séjour, à un emploi, à un logement, pour pouvoir faire venir sa fille qui se trouve dans une situation délicate. Plus les professionnels focalisent le suivi sur les difficultés de son fils, plus la mère s'oppose aux rencontres avec eux, et plus l'institution raccourcit le séjour, aboutissant à une sortie dans des conditions instables, sans soutien et sans relais possible, alors que l'enfant est repéré comme étant en difficulté.

Il existe ainsi un lien entre inquiétudes sur le développement de l'enfant, relation difficile entre la mère et les professionnels, et sortie conflictuelle sans adresse : les enfants les plus en difficulté ont finalement davantage de risque de sortir dans l'environnement le moins stable. On retrouve des dynamiques similaires dans les deux départements et chez tous les 
profils de femmes accueillies. Elles mettent au jour le paradoxe de l'aide contrainte, et d'un accompagnement focalisé sur la relation mère-enfant mis en œuvre dans un lieu d'hébergement. Plus les professionnels sentent les mères résistantes à l'intervention éducative, plus ils pointent les difficultés de l'enfant, et plus les positions se rigidifient de part et d'autre. À ce titre, il est possible d'identifier un lien entre les dynamiques observables en cours d'accompagnement et les processus de construction des parcours, au moment de la sortie et ensuite, sans pour autant que ce parcours soit réductible à une mesure de l'impact de l'intervention.

\section{Conclusion}

L'approche séquentielle permet ainsi de redéfinir et d'expliciter les liens que l'on fait entre parcours de vie et interventions sociales. Si les recherches menées en sciences de l'éducation visent régulièrement un objectif d'utilité et de transformation sociale, elles doivent pour cela pouvoir disposer d'outils et de cadres conceptuels permettant de penser les liens entre parcours de vie et interventions. L'ambition de décrire la portée des processus observés et leurs ingrédients au cours de la dynamique temporelle ne doit pas conduire à s'enfermer dans le piège de l'assignation causale réductrice de la réalité sociale et de l'épaisseur des processus observés. En effet, si la clôture du système causal ne pourra jamais être garantie dans ces situations réelles, d'un point de vue éthique, il est également important de trouver des outils qui donnent à lire et comprendre des processus à grande échelle, sans en réduire la complexité.

Est-ce à dire que nous plaidons pour une posture uniquement objectivante ? Que parce que nous définissons de manière extérieure les dimensions pertinentes de la trajectoire, l'acteur n'a pas sa place ? Non, au contraire. À rebours d'une approche épidémiologique, l'approche séquentielle essaye au contraire de mettre en lumière les ingrédients des bifurcations et de résoudre ainsi le paradoxe selon lequel les moments que l'on peut expliquer causalement sont moins significatifs pour l'acteur que les moments inexplicables causalement. En ce sens, l'approche séquentielle se complète bien par une perspective subjective, davantage d'ailleurs que les approches épidémiologiques qui s'intéresseront peu au sens donné par les acteurs. C'est en cela que cette approche des processus nous parait pertinente et porteuse pour l'analyse des parcours pendant ou après une intervention : elle permet de comprendre les ingrédients des processus et les enchaînements d'événements, sans s'enfermer dans une recherche des causes, souvent insoluble et également démobilisatrice pour les personnes accompagnées et pour les professionnels. 